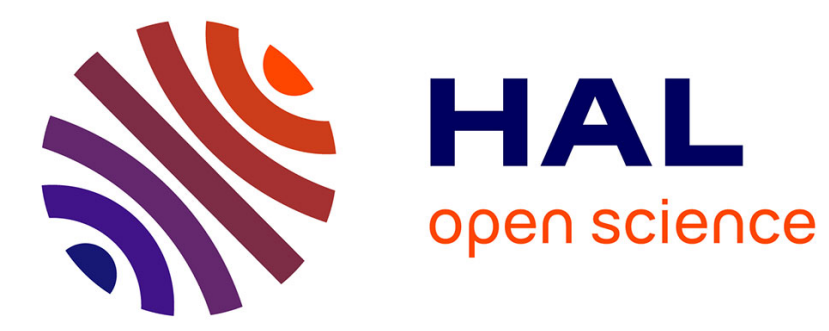

\title{
Optimized Silica Shell Synthesis Surrounding Gold Nanorods for Enhanced Spectroscopies
}

Marina Santana Vega, François Brisset, Guillaume Laurent

\section{To cite this version:}

Marina Santana Vega, François Brisset, Guillaume Laurent. Optimized Silica Shell Synthesis Surrounding Gold Nanorods for Enhanced Spectroscopies. Plasmonics, 2020, 10.1007/s11468-020-013322. hal-03065976

\section{HAL Id: hal-03065976 https://hal.science/hal-03065976}

Submitted on 23 Nov 2021

HAL is a multi-disciplinary open access archive for the deposit and dissemination of scientific research documents, whether they are published or not. The documents may come from teaching and research institutions in France or abroad, or from public or private research centers.
L'archive ouverte pluridisciplinaire HAL, est destinée au dépôt et à la diffusion de documents scientifiques de niveau recherche, publiés ou non, émanant des établissements d'enseignement et de recherche français ou étrangers, des laboratoires publics ou privés. 


\title{
Optimized silica shell synthesis surrounding gold nanorods for enhanced spectroscopies
}

\author{
Marina Santana Vega ${ }^{\dagger}$, François Brisset ${ }^{\ddagger}$ and Guillaume Laurent ${ }^{*}, \dagger$ \\ † Université Paris-Saclay, ENS Paris-Saclay, CNRS, PPSM, 91190 Gif-sur-Yvette, France. \\ E-mail: guillaume.laurent@ens-cachan.fr \\ + ICMMO, SP2M, Université Paris-Saclay, UMR CNRS 8182, 91405, Orsay Cedex, France.
}

\begin{abstract}
We present a precise study for coating gold nanorods (GNRs) by a silica shell layer. Different parameters like $\mathrm{pH}$, reaction time and, most importantly, the ratio between the selected surfactant and the tetraethyl orthosilicate (TEOS) are studied and discussed. This latter is the key parameter to increase reproducibility, stability, reaction time and the control of the targeted silica shell thickness. Moreover, the silica shell growth induces an optical shift of the localized surface plasmon resonance (LSPR) bands, which is explained by the change of the local refractive index. Both extinction spectroscopy and scanning electronic microscopy (SEM) are used to characterize the growth of the silica shell in time. Based on these results, it is thus possible to follow the growth process just by extinction spectroscopy, which ensures a rapid control of the reaction and makes it possible to stop it on time depending on the targeted shell thickness. Controlling the thickness is mandatory for further GNRs functionalization and surface plasmon enhanced spectroscopies.
\end{abstract}

\section{Keywords}

Gold nanorods. Silica Shell synthesis. Core-shell materials. Spacer thickness control. Shell growth spectrometry monitoring

\section{Introduction}

The design and development of new hybrid and functional systems are very important in field of materials science due to specific limitations such as the use and manipulation of colloidal particles (i.e., low stability, high chemical reactivity, and non-desired aggregation processes). To overcome some of these limitations, synthesis of core-shell structures using "bottom-up" approach shows many advantages. Silica for coating of colloidal particles, polymers, and surfactant aggregates have been widely used, especially for enhancement of colloidal stability.[1] More precisely, concerning colloidal systems, many different ones have been studied, such as metallic nanoparticles, semiconductors, magnetic, ceramic nanoparticles and graphene coated with gold.[2-6]

Using silica for coating material relies in its high stability, especially in aqueous media, but also on the easy regulation of the coating process, chemical inertness, controlled porosity, adaptability and optical transparency. Mainly two factors explain this stability. [7] The first one is that van der Waals interactions are much lower than those involving other nanoparticles. The second one is that cations and positively charged molecules can be tightly attached to the silica layer at silica-water interfaces under basic conditions. The consequence is that using silica shell offers steric and electrostatic protection at the same time, which prevents from aggregation. Finally, silica is also a low-cost material that can modify the surface of nanoparticles in terms of functionalization and biocompatibility, which opens the way for specific applications like enhanced spectroscopies, diagnostic and therapeutic technics.

A number of reports have been devoted to silica coating of colloidal particles by several aqueous classical methods.[8] Nevertheless, this work focuses on the Stöber process, which was first discovered in 1968 by Werner Stöber.[9] Although nowadays it is frequently used in core-shell morphologies, it was firstly developed as a physical chemistry process for the generation of monodisperse particles of silica. The formation of silica takes place through the hydrolysis and condensation of alkyl silicates under alkaline conditions in alcoholic solutions. As explained before, due to Localized Surface Plasmon Resonance (LSPR), gold nanorods (GNRs) are suitable candidates for a wide variety of applications. However, the poor stability of these systems when removing the coating surfactants, becomes a problem for their applications in many fields. The employment of 
silica coating process, provides high stability and chemical inertness to the particles. Silica shells also act as surface bases for ligand exchange and functionalization of the particles using click chemistry.[10]

Silica coated gold nanorods are usually prepared by a process of hydrolysis and condensation of tetraethyl orthosilicate (TEOS) in a water alcohol mixture. Several methods of synthesis have been reported in the recent years. In all the cases TEOS is used as the source of silica. Although TEOS must be added in ethanol or methanol, the reaction, in all cases, takes place in a water-rich system rather than in alcohol-rich system. Indeed, the dielectric constant of these alcohols is much smaller than that of water, therefore, these mediums provide less electric shielding, causing the aggregation of the particles.[11] The successful silica coating depends on several parameters, including the ratio of [surfactant]/[TEOS], $\mathrm{pH}$ and aging time.[12]

In this paper, hexadecyl(trimethyl)ammonium bromide (CTAB) is used as the main surfactant for both the GNRs synthesis and the silica shell coating. In first place, extra CTAB is added to the original GNRs solution in order to prevent the aggregation of GNRs upon the addition of TEOS. Furthermore, this surfactant forms soluble micelles acting as templates and carriers for silica sol in solution. This is why the concentration of CTAB must be higher than the critical micelle concentration in water $(0.002 \mathrm{M})$. Secondly, the adsorption of the silica sols is driven by electrostatic attraction between the cationic surfactant (ammonium group of CTAB, positively charged) and the silica sols (negatively charged), so it is necessary to keep the $\mathrm{pH}$ around 10 in order to control the hydrolysis and adsorption of TEOS. For this purpose, ammonia salts [13] or $\mathrm{NaOH}$ are used. Finally, the aging time of the reaction must be chosen according to the other parameters. Different ratios of [CTAB]/[TEOS] result in different silica thickness for the same reaction time.

\section{Experimental}

Based on several methods described in the literature, one successful protocol has been developed through this project. [14-18] We found the correct synthesis parameters to perform stable and reproducible silica-coated gold nanorods with great control of the silica shell thickness.

First of all, gold nanorods with specific aspect ratio were prepared by a modified "seed mediated growth method" using binary surfactant mixtures. In this synthesis, procedure sodium oleate $(\mathrm{NaOL})$ is used in combination with CTAB in order to achieve greater tunability of GNRs dimensions. This anionic surfactant has a double bond that allows the reduction of the gold salt in the absence of other reducers, so a lower molar ratio of gold:ascorbic acid (AA) is used, compared to the original method. The mixture of CTAB and NaOL also allows the use of lower concentration of surfactant in the reaction.[19]

The reaction has two steps: First, a small quantity of gold salt is reduced using a strong reducing agent as sodium borohydride in order to create small gold nanospheres or "seeds", which will be used as cores of the gold nanorods. Secondly, a growth solution provides the gold salt and the other reactants for the growth of these seeds with the desired size and shape. These reactions were prepared as follows:

Seed Solution: $5 \mathrm{~mL}$ of $0.5 \mathrm{mM}$ HAuCl 4 was mixed with $5 \mathrm{~mL}$ of $0.2 \mathrm{M} \mathrm{CTAB}$ solution in a beaker. $0.6 \mathrm{~mL}$ of fresh $\mathrm{NaBH} 4$ was diluted to $1 \mathrm{~mL}$ with water and was then injected to the $\mathrm{Au}(\mathrm{III})$-CTAB solution under vigorous stirring. The solution color changed from yellow to brownish yellow and the stirring was stopped after $2 \mathrm{~min}$. The seed solution was aged at room temperature for (at least) $30 \mathrm{~min}$ before use.

Growth solution: A certain quantity of $\mathrm{CTAB}$ and $\mathrm{NaOL}$ were dissolved in $125 \mathrm{~mL}$ of water in a $0.5 \mathrm{~L}$ Erlenmeyer flask. $4 \mathrm{mM} \mathrm{AgNO} 3$ solution was added. The mixture was kept undisturbed at $30^{\circ} \mathrm{C}$ for $15 \mathrm{~min}$ after which $125 \mathrm{~mL}$ of $1 \mathrm{mM} \mathrm{HAuCl} 4$ solution was added. The solution became colorless after $90 \mathrm{~min}$ of stirring (700 rpm) and a certain volume of $\mathrm{HCl}(37 \mathrm{wt} . \%$ in water, $12.1 \mathrm{M})$ was then introduced to adjust the $\mathrm{pH}$. After another $15 \mathrm{~min}$ of slow stirring ( $400 \mathrm{rpm}), 0.6 \mathrm{~mL}$ of $0.064 \mathrm{M}$ ascorbic acid (AA) was added and the solution was vigorously stirred for $30 \mathrm{~s}$. Finally, a small amount of seed solution was injected into the growth solution. The resultant mixture was stirred for $30 \mathrm{~s}$ and left undisturbed at $30^{\circ} \mathrm{C}$ for $12-15 \mathrm{~h}$ for GNRs growth. The final products were isolated by centrifugation at $6000 \mathrm{rpm}$ for $20 \mathrm{~min}$ followed by removal of the supernatant. Precise synthesis parameters, optical and structural characterizations were done in supporting information (Table and Figure S1).

Secondly, two different synthesis methods were used for coating the gold nanorods prepared previously with a silica shell using the modified Stöber protocol.

Procedure A: Several $\mathrm{mL}$ of GNR were washed by centrifugation during $20 \mathrm{~min}$ at $6000 \mathrm{rpm}$. The supernatant was removed and the pellet was dispersed in the same quantity of water. Then $0.1 \mathrm{M} \mathrm{NaOH}$ was added under vigorous stirring in order to keep the $\mathrm{pH}$ around 10. A certain quantity of solution of TEOS in $\mathrm{MeOH}(20 \% \mathrm{v} / \mathrm{v})$ 
was then added under gentle stirring. The final concentration of TEOS was $0.018 \mathrm{M}$. The reaction was stopped by centrifugation during 10-20 min at $6000 \mathrm{rpm}$ after different chosen times. The pellet was dispersed in the same quantity of water.

Procedure B: Several $\mathrm{mL}$ of GNR were washed by centrifugation during $20 \mathrm{~min}$ at $6000 \mathrm{rpm}$ twice to remove as much $\mathrm{CTAB}$ as possible. The supernatant was removed and the pellet was dispersed in the same quantity of water. After washing, a known concentration of $\mathrm{CTAB}$ was added to the solution. $0.1 \mathrm{M} \mathrm{NaOH}$ was added under vigorous stirring in order to keep the $\mathrm{pH}$ around $10(0.01 \mathrm{eq} \mathrm{v} / \mathrm{v})$. Then, a solution of TEOS in $\mathrm{MeOH}(20 \% \mathrm{v} / \mathrm{v})$ was added under gentle stirring. The reaction was stopped by centrifugation during 15-20 min at $6000 \mathrm{rpm}$ after different chosen times. The pellet was dispersed in the same quantity of water.

\section{Results and discussion}

Silica shells with different thicknesses were successfully synthesized using both procedures. In the case of the procedure A (without controlling the CTAB/TEOS ratio), we could observe a shift in the maximum of the longitudinal plasmon resonance (Figures 1a and 1b). This red shift increased with the aging time of the reaction, until a maximum of around $4 \mathrm{~h}$ in all the experiments. Considering the transverse plasmon resonance, a smaller spectral shift is observed (Figure S2, supporting information). Opposite to the one of the longitudinal LSPR band, this spectral shift does not increase with the reaction time. For the rest of the discussion only the longitudinal LSPR band will be considered, as it is the most sensible to changes of the near-field environment. Samples were also characterized by scanning electronic microscope (SEM), to measure precisely the thickness of the silica shell (Figure 1c). However, when we tried to do these experiments again, we encountered reproducibility problems with this procedure. This was mainly due to the concentration of CTAB, which could not be precisely controlled with only one centrifugation. This is why, as described previously, an extra centrifugation was included in the procedure B. The point was to remove as much CTAB as possible to the initial solution of GNRs and, in a second step, to add a known concentration after washing the solution. By controlling the concentration of surfactant, and thus, the ratio of $\mathrm{CTAB} / \mathrm{TEOS}$, it was possible to reproduce several experiments, and to improve the tunability of the silica shell thickness. All the experimental data for procedure B are shown in the Table 1 and the corresponding extinction spectra are shown in Figure S3 (supporting information).

Using procedure $\mathrm{B}$, we firstly prepared different concentrations of CTAB, ranging from 0.001 to $0.020 \mathrm{M}$ keeping a constant concentration of TEOS of $0.005 \mathrm{M}$. The value of the critical micelle concentration (CMC) of the CTAB in water was 0.001 , but the solutions were only stable with a concentration of $0.005 \mathrm{M}$ or higher because the $\mathrm{CMC}$ was calculated for spherical micelles. In our case, the relation surface/volume was higher because of the rod shape of these micelles. Under $0.005 \mathrm{M}$ the nanoparticles were not stable enough to perform the synthesis. Over this value, higher stability was found when increasing the quantity of CTAB in the reaction. (Exp. ${ }^{\circ} 1$ to 4 in table S2)

Considering the GNRs covered with silica after $20 \mathrm{~h}$ of reaction, we conclude that using procedure B allows to obtain stable hybrid nanoparticles whatever the CTAB concentration used. When considering the observed spectral shift for the different experiments after 4 and $20 \mathrm{~h}$, no big influence of the CTAB concentration was observed, except for exp. $n^{\circ} 3$ where the CTAB/TEOS ratio was 0.2. In this case, the spectral shift was smaller and the silica shell thickness was thinner, which means that using these parameters, a longer reaction time was achieved. This observation is important because having a slower kinetic means an easier control of the silica shell thickness. So, after several experiments, we found the CTAB concentration of $0.01 \mathrm{M}$ as a good compromise for the synthesis. $\left(\operatorname{Exp~n}^{\circ} 3\right)$

Secondly, different concentrations of TEOS were also checked, by keeping a constant concentration of CTAB of $0.005 \mathrm{M}$ and $0.010 \mathrm{M}$, and using concentrations of TEOS between 0.017 and $0.050 \mathrm{M}$. (Exp n 1,3 and 5 to 7 ) With a TEOS concentration of $0.017 \mathrm{M}$ and $0.028 \mathrm{M}$, the maximum spectral shift was mainly observed after $4 \mathrm{~h}$ of reaction time. This means that the reaction occurred too quickly. By increasing the TEOS concentration to $0.05 \mathrm{M}$, the reaction became slower, being optimum when using CTAB concentration $0.005 \mathrm{M}$ and $0.010 \mathrm{M}$. The most important observation to take into account is not the final spectral shift obtained after $20 \mathrm{~h}$ of reaction time but its evolution. Considering that point, it appears that the two optimal CTAB/TEOS ratios of $0.1\left(\operatorname{Exp~n}^{\circ} 1\right)$ and $0.2\left(\operatorname{Exp~}^{\circ} 3\right)$, enable a higher stability and a better control of the reaction along the time.

Precise results of experiments using CTAB/TEOS ratios of $0.1\left(\operatorname{Exp}^{\circ} 1\right)$ and $0.2\left(\operatorname{Exp}^{\circ} 3\right)$ are shown in Figure $2 a$ and $b$. More SEM measurements are shown in Figure S4 (supporting information). We can see that the silica shell thickness increased with the reaction time, which can be directly correlated to the observed spectral shift. 
Furthermore, the spectrum obtained with the second experiment, i.e., using the biggest concentration of CTAB, shows the highest reproducibility of this synthesis. The graphics in the Figure 3 show the dependence between the optical shift, the silica thickness and the reaction time. In the Figure $3 \mathrm{a}$, we can observe the linear dependence found between the shift in the longitudinal LSPR wavelength and the thickness of the silica shell. More precisely, two different behaviors corresponding to different slopes are observed emphasizing that the reaction occurs in two steps. Before $2 \mathrm{~h}$ of reaction, the silica shell started appearing, but surrounded the GNRs inhomogeneously. After $2 \mathrm{~h}$ of reaction, the silica shell completely surrounded the GNRs and then increased its thickness. The position and intensity of the LSPR bands are strongly dependent of the nanoparticles' shape, size, composition as well as the value of the refractive index in the nanoparticle vicinity.[20-23] The refractive index can be modified by targeted organic molecules, in case of biosensing applications [24], or by specific functionalization of the nanoparticle. This change can be easily detected by extinction spectroscopy. On the other hand, it is complicated to calculate precisely the value of the refractive index during the growth of the silica shell. In fact, many parameters influence its value such as the solvent, the thickness of the silica shell but also the CTAB and TEOS molecules in the vicinity of the nanoparticles. The evolution of these parameters has to be considered during the reaction time until the silica thickness reaches around $20 \mathrm{~nm}$. The value of the enhanced electromagnetic field for plasmonic nanoparticles decreases as the inverse of the cube distance from the surface, which means that the modulation of the refractive index will mainly affect the LSPR properties for distance smaller than $20 \mathrm{~nm}$.[25, 26] Looking more precisely to the silica shell functionalization, before $2 \mathrm{~h}$ of reaction the change of the local refractive index is not linear. At this moment, silica germs appear at the surface of the GNRs and the local concentration of CTAB and TEOS change at the same time. After $2 \mathrm{~h}$ of reaction, only the thickness of the silica shell influences the value of the refractive index which induces a linear modulation of the spectroscopic properties of the longitudinal LSPR band. These conclusions were the same for both CTAB/TEOS ratio, but in Figure 3b, we can see that increasing this ratio using higher CTAB concentration allows decreasing the reaction kinetic and makes the control of the targeted silica shell thickness easier.

By using protocol B with the right concentration of surfactant and TEOS, it is possible to control the reaction, and, consequently, the silica shell thickness surrounding the gold nanorods. The linear dependence between the spectral shift and the silica shell thickness allows estimating the rate of the reaction without doing SEM imaging. Furthermore, in these syntheses, thickness between 10 and $20 \mathrm{~nm}$ were achieved with a quite accurate control. Moreover, in other published silica shell protocols, controlling precisely the thickness by a two nanometer step is very difficult. In our case, by controlling precisely the CTAB/TEOS ratio, we could increase the reaction time, and thus make it easier to achieve the targeted shell thickness.

In this case, using $0.01 \mathrm{M}$ of $\mathrm{CTAB}$ resulted in a slower reaction. The reaction was stable after several hours, therefore it could be used with longer times of reaction. On the other hand, by using $0.005 \mathrm{M}$ of CTAB, a spectral shift of $9 \mathrm{~nm}$ was observed in just $4 \mathrm{~h}$, which corresponds to an estimated thickness of around $15 \mathrm{~nm}$.

\section{Conclusion}

By looking the influence of different parameters like the concentration of the surfactant and the ratio between the surfactant concentration and the tetraethyl orthosilicate concentration, different thicknesses have been reached. Optimizing these parameters, we defined a simple protocol allowing a high control of the silica thickness with the aging time of the reaction, getting satisfactory results (thickness between 10 and $20 \mathrm{~nm}$ with a $2 \mathrm{~nm}$ step) with short times of reaction (4 hours). On a second hand, the increase of the silica shell thickness clearly induces a red shift of the spectral position of the LSPR bands, which is directly attributed to the modulation of the local refractive index. Such modulation is very convenient because it allows spectrometry monitoring of the shell growth, and therefore, gives an easy way to control and stop the reaction process in order to obtain the targeted silica thickness. Moreover, this protocol presents a high reproducibility, which is very important for a possible further functionalization with other molecules by click-chemistry.[27, 28] For this application, the precise control of the distance between the metallic particle and other molecule is mandatory, such as, for example, in the case of fluorescence emission modified by surface plasmon.[29]

\section{Ethical Approval}

Not applicable 


\section{Consent to Participate}

Not applicable

\section{Consent to Publish}

Not applicable

\section{Authors Contributions}

All authors contributed to the study. Synthesis and extinction spectroscopy were performed by Marina Santana Vega; scanning electronic microscopy was performed by François Brisset; initial conception, design and analysis were performed by Guillaume Laurent. The first draft of the manuscript was written by Guillaume Laurent and all authors commented on previous versions of the manuscript. All authors read and approved the final manuscript.

\section{Funding}

Not applicable

\section{Competing Interests}

Not applicable

\section{Availability of data and materials}

Not applicable

\section{References}

[1] Guerrero-Martínez A, Pérez-Juste J, Liz-Marzán L M (2010) Advanced Materials 22: 1182

[2] Graf C, Vossen D L J, Imhof A, van Blaaderen A (2003) Langmuir 19: 6693

[3] Guo Q, Li C, Deng B, Yuan S, Guinea F, Xia F (2017) ACS Photonics 12/: 2989

[4] Wei W, Nong J, Mei Y, Zhong C, Lan G, Hu W (2018) Sensors and Actuators B: Chemical 273: 1548

[5] Wei W, Chen N, Nong J, Lan G, Wang W, Yi J, Tang L (2018) Optics Express 26: 16903

[6] Nong J, Wei W, Lan G, Luo P, Guo C, Yi J-M, Tang L (2020) Anal. Chem. 2020, https://doi.org/10.1021/acs.analchem.0c02733

[7] Shen R, Camargo P H C, Xia Y, Yang H (2008) Langmuir 25: 1189

[8] Guerrero-Martinez A, Fibikar S, Pastoriza-Santos I, Liz-Marzan L M (2009) Angew. Chemie 48: 1266

[9] Stöber W, Fink A, Bohn E (1968) Journal of Colloid and Interface Science 26: 62

[10 Liu W, Zhu Z, Deng K, Li Z, Zhou Y, Qiu H, Gao Y, Che S, Tang Z (2013) J. Am. Chem. Soc. 135: 9659

[11] Huang C C, Huang C H, Kuo I T, Chau L K,. Yang T S (2012) Colloids and Surfaces A : Physi. Eng. Aspects 409: 61

[12] Zhang J J, Liu Y G, Jiang L P, Zhu J J (2008) Electrochem. Comm. 10: 355

[13] Wu S, Schell W, Lublow M, Kaiser J, Aichele T, Schietinger S, Polzer F, Kühn S, Guo X, Benson O, Ballauff M, Lu Y (2013) Colloid Polym. Sci. 291: 585

[14] Ohmori M, Matijevic E (1993) J. Colloid Interface Sci. 160: 288

[15] Liz-Marzan L M, Philipse A P (1995) J. Colloid Interface Sci. 176: 459

[16] Plueddermann E P (1991) Silane Coupling Agents. Plenum Press, New York

[17] Liz-Marzan L M, Giersig M, Mulvaney P (1996) Chem. Commun. 731

[18] Liz-Marzan L M, Giersig M, Mulvaney P (1996) Langmuir 12: 4329

[19] Ye X, Zheng C, Chen J, Gao Y, Murray C B (2014) Nanoletters 13: 765

[20] Mayer K M, Hafner J H (2011) Chem. Rev.111: 3828

[21] Willets K A, Van Duyne R P (2007) Ann. Rev. Phys. Chem. 58: 267

[22] Homola J, Yeea S S, Gauglitz G (1999) Sens. Actuators B Chem. 54: 3

[23] Schasfoort R B M, Tudos A J (2008) Handbook of Surface Plasmon Resonance (Royal Society of Chemistry)

[24] Nusz G J, Curry A C, Marinakos S M, Wax A, Chilkoti A (2009) ACS Nano 3: 795

[25] Reineck P, Gomez D, Hock Ng S, Karg M, Bell T, Mulvaney M, Bach U (2013) ACS Nano 7: 6636

[26] Anger P, Bharadwaj P, Novotny L (2006) Phys. Rev. Lett. 96: 113002

[27] Abadeer N S, Brennan M R, Wilson W L, Murphy C J (2014) ACS Nano 8: 8392

[28] Ouhenia-Ouadahi K, Yasukuni R, Yu P, Laurent G, Pavageau C, Grand J, Guérin J, Léaustic A, Félidj N, Aubard J, Nakatani K, Métivier R (2014) Chem. Commun. 50: 7299 
[29] Gavilán-Rubio H, Coelho J P, González-Rubio G, Tardajos G, Osío Barcina J, Salgado C, GuerreroMartínez A (2016) Israel Journal of Chemistry 56: 249 

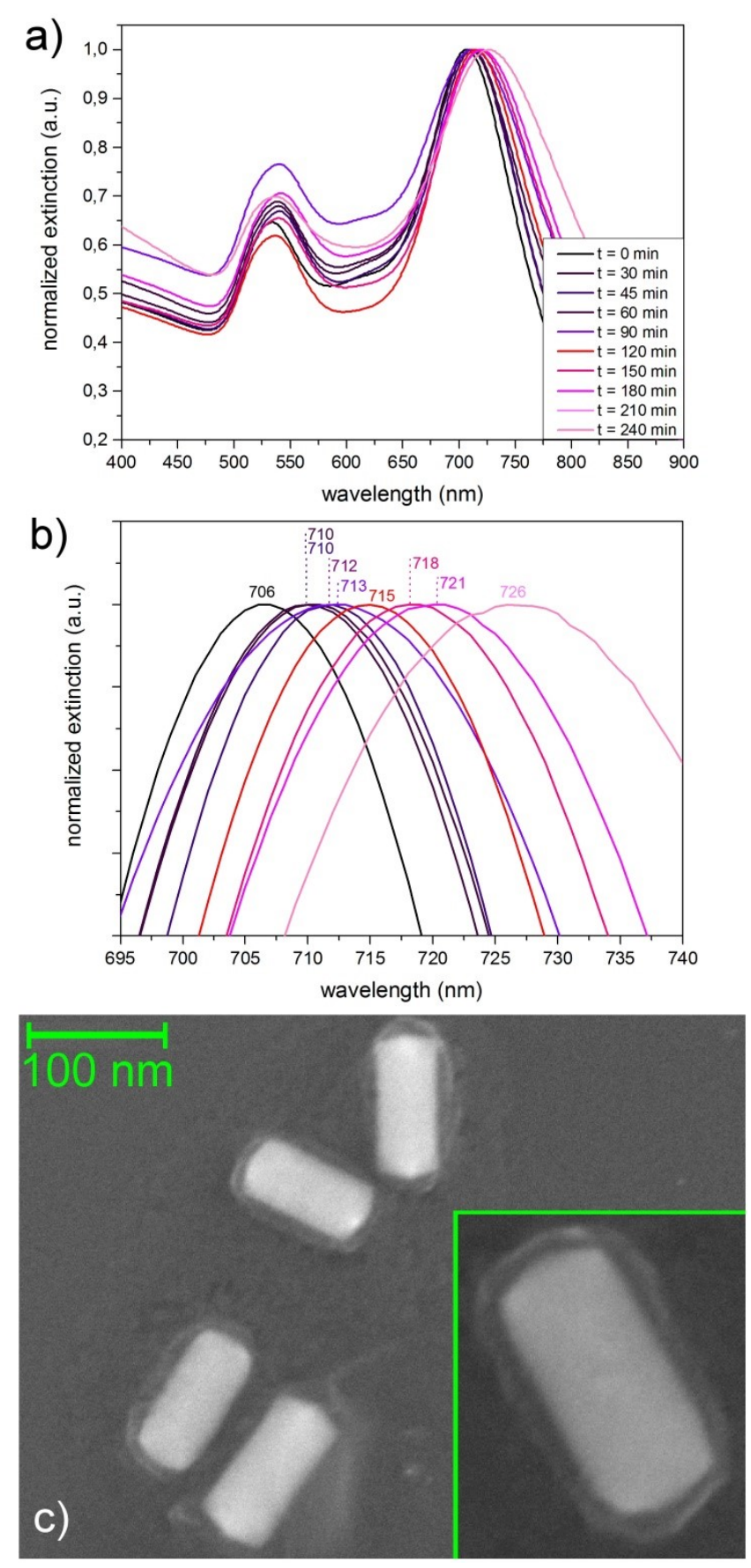

Figure 1. a) Extinction spectra of gold nanorods covered by silica shell. Each spectrum corresponds to different reaction time ; b) zoom of part a) showing the spectral red shift of the longitudinal LSPR band due to the increase of the silica shell thickness ; c) SEM image showing the silica shell after 240 min of reaction time. Scale bar represents $100 \mathrm{~nm}$. 

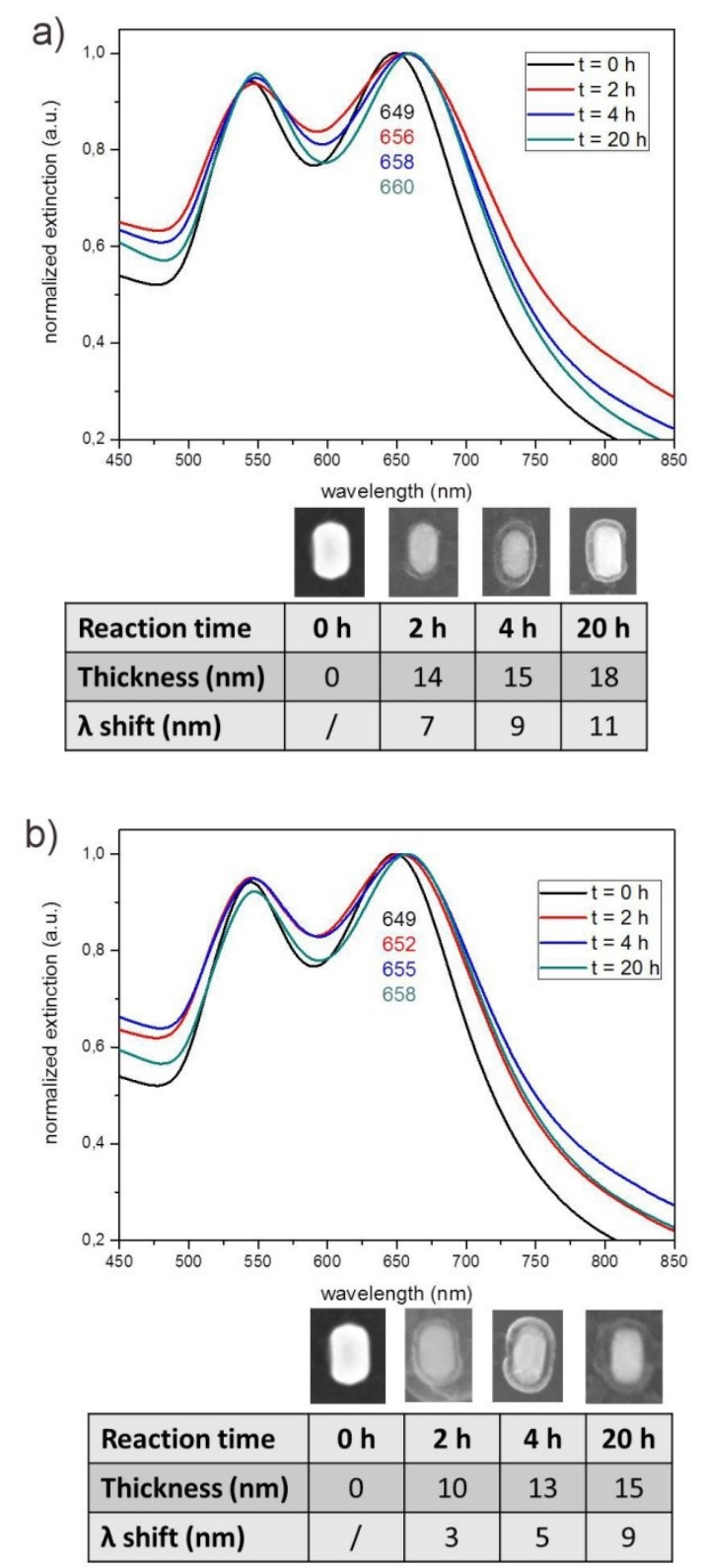

Figure 2. Extinction spectra of gold nanorods covered by silica shell after different reaction time. The table shows the silica shell thickness measured by SEM imaging and the spectral shift for different reaction time. $\mathrm{CTAB} / \mathrm{TEOS}$ ratio is 0.1 for a) and 0.2 for $b$ ). 

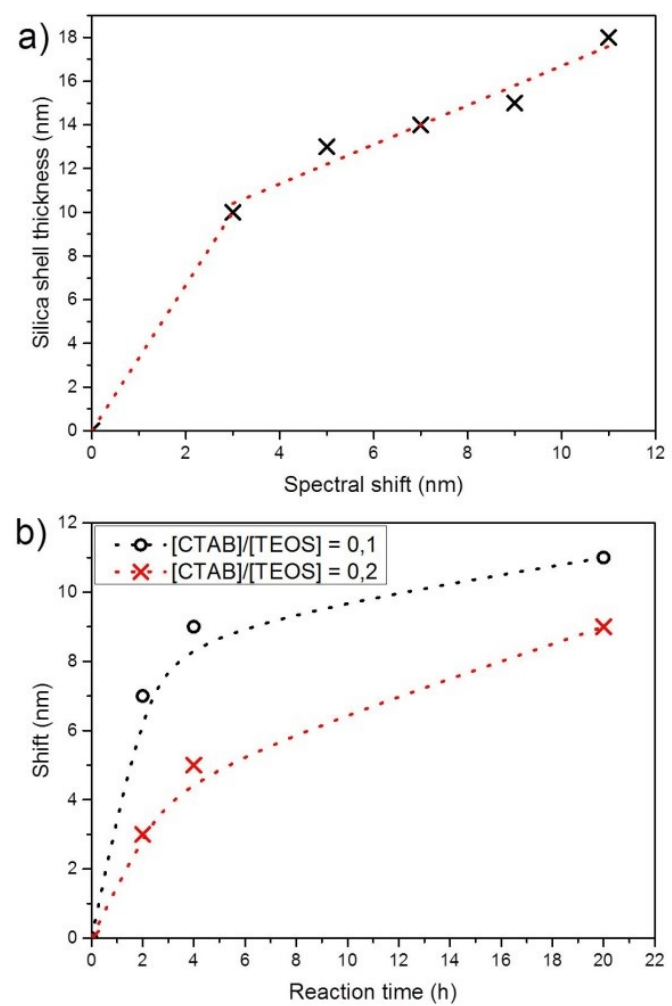

Figure 3. a) relation between the silica shell thickness and the optical spectral shift observed in extinction spectra ; b) evolution of the optical spectral shift with the reaction time. Dotted lines are just guidelines. 


\begin{tabular}{|c|c|c|c|c|c|}
\hline Exp. $\mathrm{n}^{\circ}$ & {$[\mathrm{CTAB}]\left(\mathrm{mol} .1^{-1}\right)$} & {$[\mathrm{TEOS}]\left(\mathrm{mol} . \mathrm{l}^{-1}\right)$} & {$[\mathrm{CTAB}] /[\mathrm{TEOS}]$} & \multicolumn{2}{|c|}{ Spectral shift $(\mathrm{nm})$} \\
\cline { 5 - 6 } & & & & after 4 hours & after 20 hours \\
\hline 1 & 0,005 & 0,05 & 0,1 & 9 & 11 \\
\hline 2 & 0,0075 & 0,05 & 0,15 & 9 & 13 \\
\hline 3 & 0,01 & 0,05 & 0,2 & 5 & 9 \\
\hline 4 & 0,02 & 0,05 & 0,4 & 9 & 12 \\
\hline 5 & 0,005 & 0,028 & 0,18 & 12 & 12 \\
\hline 6 & 0,01 & 0,028 & 0,36 & 9 & 10 \\
\hline 7 & 0,01 & 0,017 & 0,59 & 7 & 8 \\
\hline
\end{tabular}

Table 1. Synthesis parameters for the silica shell. Gold nanorods B are used with the procedure B 